\title{
Anticipatory changes in state, dual-process theory, and preliminary OR processes
}

\author{
ROBERT J. BARRY \\ University of New South Wales, Kensington, Australia
}

\begin{abstract}
Recent studies of phasic response fractionation in the OR context have led to a theoretical development of the multiregister model of Barry (1979), which emphasizes the role of preliminary processes in OR elicitation. In this new model (e.g., Barry, 1981b), the novelty and magnitude of the stimulus are coded in separate registers which are similar in their operating characteristics to the hypothetical processes $\mathrm{H}$ and $\mathrm{S}$ of dual-process theory (Groves \& Thompson, 1970), and which interact to produce the OR. The present study examined the parallel between $\mathrm{S}$ and the stimulus magnitude register by manipulating anticipatory cardiac arousal between stimuli. A warned target stimulus was predicted to provide a context of enhanced cardiac arousal in comparison with a warned nontarget. The predicted S effect was obtained in the peripheral pulse amplitude response (PPAR), an index of the magnitude register. This and other results of response fractionation support the new model of preliminary OR processes. The results also bear upon recent findings of inconsistent habituation with PPAR measures.
\end{abstract}

Sokolov's conceptualization of the orienting response (OR) as "a complex reaction of the whole organism" (1955, p. 134) and "a unitary system" $(1960$, p. 191), consistently reflected in his references to "the OR" in the singular sense, requires that similar stimulus-response relationships will hold for all OR indicators. The most commonly used physiological indices from those listed by Sokolov, and which have been of special interest in this laboratory, are the GSR, vascular reactions of the head and hand, respiratory and heart rate changes, and EEG alpha desynchronization. In recent years, research using such indices has clearly identified problems with the unitary OR theory (e.g., Furedy \& Arabian, 1979; Ginsberg \& Furedy, 1974). However, the common approach has been to express reservations about the sensitivity of "misbehaving" indicators rather than about the unitary construct itself. In contrast, our efforts have been concentrated upon the search for other regularizing principles compatible with such apparent misbehavior.

A number of studies which failed to find the predicted covariation led Barry $(1978,1979)$ to propose an alternative four-system structure to accommodate the observed fractionation of responses under differ-

A preliminary version of this paper was presented at the 21st Annual Meeting of the Society for Psychophysiological Research in Washington, D.C., in October 1981. The assistance of Angela L. James in the collection and analysis of the data is gratefully acknowledged. The paper was written while the author was on sabbatical leave in the Department of Psychology at UCLA, and his thanks are extended to Irving Maltzman for his critical comments on an earlier version of the manuscript. Address reprint requests to Robert J. Barry, School of Education, University of New South Wales, Kensington, N.S.W. 2033, Australia. ent stimulus manipulations. Further empirical and theoretical development (Barry, 1981b, 1982; Barry \& James, 1981a, 1981b) led to a new model of preliminary processes in OR elicitation, which is illustrated in Figure 1. In this model, stimulus occurrence is coded in the stimulus register, producing an evoked cardiac response (ECR1) of heart rate (HR) deceleration and a cephalic pulse amplitude response (CPAR) of vasodilation. The novelty and magnitude of the stimulus are coded in separate registers, whose activity is reflected in respiratory pause and EEG alpha desynchronization, and in the peripheral pulse amplitude response (PPAR) of vasoconstriction, respectively. The processes underlying these registers are hypothesized to interact to produce what is conceptualized as Sokolov's functional OR, indexed by the GSR and affecting learning and perception. If

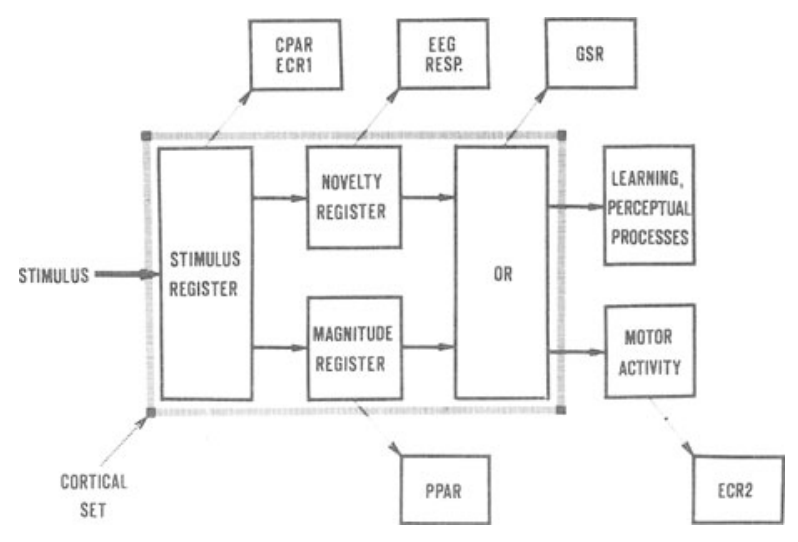

Figure 1. The Barry model of preliminary processes in OR elicitation. 
motor activity is elicited by the stimulus, as occurs in a reaction-time task, a longer latency cardiac response of heart rate acceleration (ECR2) is hypothesized to mask ECR1. This entire process of OR elicitation is "steered" by cortical set (Maltzman, 1979).

Barry and James (1981b) examined a number of parallels between the elements of dual-process theory (Groves \& Thompson, 1970) and some elements of this multiregister system. Respiratory pause (from the novelty register) exhibited many characteristics of the hypothetical $H$ process of Groves and Thompson, while PPAR (from the stimulus magnitude register) showed some similarity to their S process. These parallels were confirmed in Barry and James (1981a), so that a tentative integration of dualprocess theory and response fractionation theory was proposed by Barry (1981b). Thus, the processes involved in the novelty and magnitude registers in Figure 1 are hypothesized to show operating characteristics similar to $\mathrm{H}$ and $\mathrm{S}$, respectively, and to interact in OR production according to dual-process theory. Thus, the GSR, as an OR index, should reflect the activity of both the novelty and magnitude registers, which are separately indexed by respiratory pause and PPAR (respectively). These last two indices are hypothesized to parallel $\mathrm{H}$ and $\mathrm{S}$ in their functional characteristics.

Although dual-process theory has its most obvious relevance in the prediction of OR outcomes produced by indifferent stimuli, its potential in some studies of significance should not be overlooked. Specifically, dual-process theory provides a defined role, in terms of $S$, for the effect of state variables in OR elicitation. This aspect of dual-process theory is the focus of the present study. The work of the Laceys (e.g., Lacey \& Lacey, 1958) made the concept of arousal as a unidimensional construct as difficult to defend as the unitary OR now appears to be. However, Groves and Thompson (1970) and Thompson, Berry, Rinaldi, and Berger (1979), while clearly recognizing this problem, have argued for the general utility of such a term as a shorthand descriptor of those tonic excitatory influences, not necessarily of a unitary nature, which can affect response outcome. O'Gorman (1979) attributed all significance effects in OR elicitation to diffuse arousal differences, a position which Maltzman (1979) contradicted from previous work (Maltzman \& Raskin, 1965). Barry (1981b) found significance effects in the GSR (but not in PPAR); these were independent of cardiac arousal levels, empirical evidence confirming the stance of Maltzman (1979) and specifically contradicting O'Gorman's thesis.

While this evidence indicates that significance effects are not necessarily associated with state changes, it is possible to envision situations in which such an association between significant stimuli and differential arousal might occur. In such circumstances, the model shown in Figure 1 would predict changes in PPAR (indexing $S$ ) reflecting such mediation (since $S$ reflects the state of the organism) and a consequent effect upon the output OR as indexed by the GSR. In contrast, because $H$ is relatively state-independent, no differential outcomes of the novelty register process are expected. The existence of these various effects would support the integrated theory of preliminary processes in OR elicitation and clarify some of the determinants of significance effects in the OR. In the experiment described here, the anticipation of a target stimulus, whose occurrence was reliably signaled by a warning stimulus, was predicted to provide a situation in which arousal level would so increase. Thus, the target was predicted to produce enhanced PPARs as well as GSRs, in a context of increased cardiac arousal, when compared with a signaled nontarget.

Apart from this major prediction, other predictions may be derived from the model of preliminary processes in OR elicitation. Respiratory pause, from the novelty register, should show habituation over trials, with no differences between responses to different stimulus types. The deceleratory ECR should similarly fail to reflect stimulus type but, in addition, should not habituate with stimulus repetition. Although this is contrary to the position of Graham and Clifton (1966), Barry and Maltzman (Note 1) provide an extensive examination of this problem, and it will not be discussed here. It should be noted that dual-process theory and Sokolov's theory make similar predictions for the OR in this study, but that Sokolov's theory does not refer to preliminary processes of the type considered here.

\section{METHOD}

\section{Subjects}

Thirty-two students, aged between 20 and 25 years, volunteered to participate in the experiment to fulfill a requirement of a course in Educational Psychology at the University of New South Wales. There were 26 males and 6 females.

\section{Apparatus and Procedure}

Forty visual stimuli, consisting of uppercase letter strings of two varieties, were generated by an Exidy microcomputer. Warning signals were of the form AAAAAAAAAA or BBBBBBBBBB (10 As or 10Bs), while imperative stimuli were of the form ABAABAABBB (10 mixed As and Bs), each containing 4, 5, or 6 As. Half the imperative strings started with $A$ and contained a mean number of 5 As. The other 10 imperative strings were formed from these by replacing As with Bs and Bs with As. These 20 imperative strings were mixed randomly, except that runs of similar strings longer than 3 were not permitted. Each was then preceded by a warning signal whose letter type matched the initial letter of the imperative string. Thus, sequences of stimuli of the form AAAAAAAAAA, ABAABAABAB, BBBBBBBBBB, BBAAABABAB, . . . were prepared. Imperative signals were either target or nontarget stimuli. The subjects had to count As (or Bs) in the targets and could ignore the nontargets. The target warning stimulus matched the target letter for a given subject. The subjects were balanced over target letter and whether the target or nontarget was presented first. For a subject given $\mathbf{A}$ as the target 
letter, the sample sequence shown above would constitute: a warning of the following target (WT), the target itself (T), a warning of the following nontarget (WNT), and the nontarget (NT). For a subject whose target letter was $B$, the same sequence would constitute: WNT, NT, WT, T. Thus, each subject was presented with 10 of each of these four stimulus types. Imperative stimuli always followed the warning stimuli, but the target/nontarget stimulus pairs were randomly presented. This design allowed the investigation of significance effects, associated with 10 trials of each of these four stimulus types, independently of physical stimulus characteristics and novelty.

Stimuli were presented by the microcomputer on a Sony video monitor with a $22.5 \times 17.5 \mathrm{~cm}$ screen. Each letter was approximately $5 \times 3 \mathrm{~mm}$, and the string was centered upon the screen. Stimulus duration was $2.0 \mathrm{sec}$, with interstimulus intervals randomly varying among 40,50, and $60 \mathrm{sec}$ independently of stimulus type.

The ac-coupled skin resistance was recorded from stainless steel electrodes on the volar surfaces of the first and third fingers of the subject's left hand. Electrode area was $3 \times 2 \mathrm{~cm}$ and Biocom Inc. Biogel was used as the contact medium. ${ }^{1}$ PPA was recorded from a Biocom piezoelectric pulse pickup attached to the index finger of the subject's right hand, which rested on the arm of the subject chair. Respiration was recorded from a Stoelting bellows pneumograph attached around the subject's chest. These three channels were recorded on a Stoelting Multiscribe with a paper speed of $2.5 \mathrm{~mm} / \mathrm{sec}$, and stimulus events were recorded on a fourth channel. PPA and stimulus events were also recorded on FM tape for later computer analysis of HR and PPAR. This utilized software detection from the analog PPA record by an Interdata 6/16 with A/D.

The subjects were seated in a darkened sound-reduced room separate from the recording equipment. They sat in a comfortable armchair approximately $70 \mathrm{~cm}$ in front of the video monitor. After fitting the physiological transducers, the general nature of the task was explained. Practice stimuli, with further explanatory information, were presented on the video monitor until the subject clearly understood the task; at least two practice stimuli of each type were presented. The subject was asked to keep a running total of the number of target letters to report at the end of the experiment. ${ }^{2}$

\section{RESULTS}

For each of the 40 stimuli, a measure of prestimulus cardiac arousal and four phasic response measures were obtained from each subject. For each of these five variables separately, values were subjected to a two-way repeated-measures ANOVA, following Poor (1973), which examined the main effects of trial and stimulus type, and their interaction, on the variable. Within the 10 trials, only linear and quadratic trends were analyzed. Within stimulus type, planned orthogonal comparisons tested T against WT, NT against WNT, and (WT+T) against (WNT +NT). The first two of these comparisons should reflect anticipatory changes following the warning signals, while the third compares the relative activity (for both the warning and imperative stimuli) between the target and nontarget contexts. In presenting the results of these analyses graphically, blocks of two trials have been used to clarify trials effects.

\section{Cardiac Arousal}

The HR of the immediately prestimulus cardiac interval was taken as the cardiac arousal level for
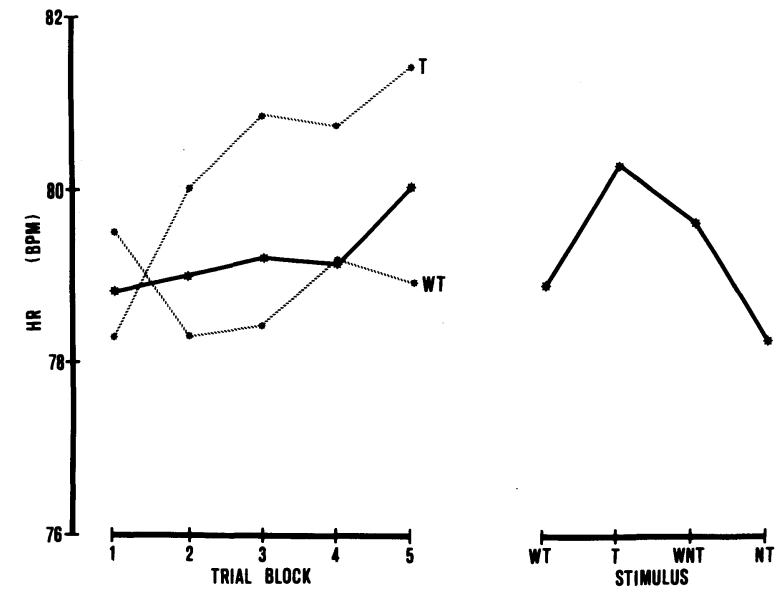

Figure 2. Prestimulus cardiac arousal as a function of trial block and stimulus type. In all figures, solid lines show main effects, while significant interactions are shown by labeled broken lines.

each stimulus. Mean values of $\mathrm{HR}$ are shown as a function of stimulus type and trial block in Figure 2. There was no significant ${ }^{3}$ overall trials effect $[F(1,31)$ $=1.56, \mathrm{MSe}=120.31$ for linear trend, and $F(1,31)$ $=.45, \mathrm{MSe}=49.26$ for quadratic trend]. The predicted increase in HR before the target stimulus was obtained $[\mathrm{F}(1,31)=5.07, \mathrm{MSe}=61.83]$, as was a decrease before the nontarget $[\mathrm{F}(1,31)=7.27, \mathrm{MSe}=$ 41.62]. There was also an interaction between the first of these effects and linear trend $[F(1,31)=4.82$, $\mathrm{MSe}=30.14]$, indicating that the anticipatory arousal increase developed with trials. No other effects approached significance.

\section{ECR}

Following previous usage in this laboratory, the ECR was defined as the largest percentage decrease, relative to prestimulus $H R$, to occur within the first 5 poststimulus beats. Thus, the slowest HR in the first 5 poststimulus beats was subtracted from that of the prestimulus cardiac interval. This difference was then expressed as a percentage of the prestimulus cardiac level defined above. These response values were submitted to an ANOVA similar to that described above. There was some increase in ECR over trials, with the linear trend over trials approaching significance $[F(1,31)=3.93, M S e=80.81$, cf. critical $F=$ 4.16]. No other effects approached significance. These results are displayed in Figure 3.

\section{Respiratory Pause}

The percentage increase in the period of the respiratory cycle containing stimulus onset compared with the preceding cycle was defined as the respiratory pause measure. This followed previous usage in this laboratory, allowing comparability of results. Mean values of this response are displayed against trial block and condition in Figure 4. There was marked habituation reflected in both linear $[F(1,31)=29.89$, 

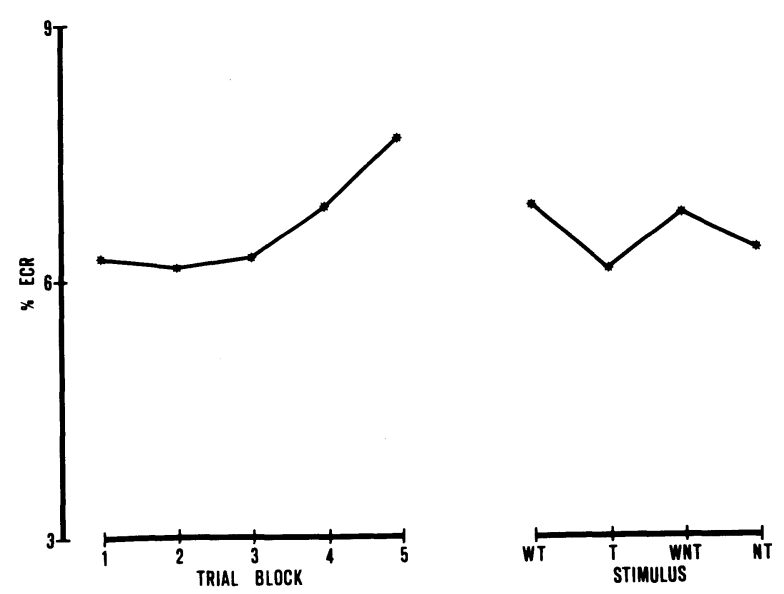

Figure 3. The evoked cardiac response (percent HR change) over trial blocks and stimulus type.
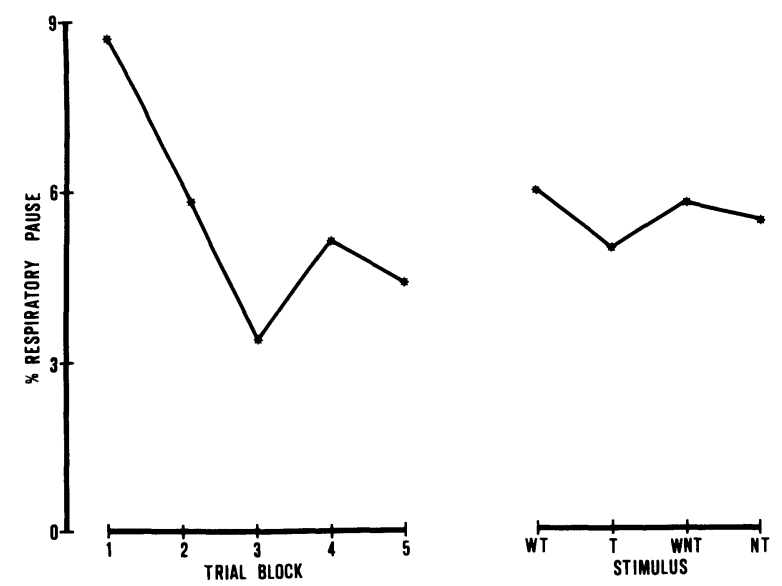

Figure 4. Respiratory pause as a function of trial block and stimulus type.

$\mathrm{MSe}=90.44]$ and quadratic $[\mathrm{F}(1,31)=9.42, \mathrm{MSe}=$ 194.86] trends over trials. No other effects were apparent.

\section{PPAR}

The smallest pulse occurring between poststimulus beats 7 and 12 was taken as the point of maximum response and used to calculate the percentage decrease in pulse amplitude from the prestimulus pulse, following previous usage in this laboratory. Figure 5 displays mean values of this response as functions of trial block and stimulus condition. A similar ANOVA to that used above indicated that, while there were no differences between responses to the warning and imperative signals for either targets or nontargets, there were larger responses within the target context (i.e., WT +T) than within the nontarget context (i.e., $\mathrm{WNT}+\mathrm{NT})[\mathrm{F}(1,31)=12.87, \mathrm{MSe}=499.12]$. There was also a general increase in PPAR over trials, indicated by a significant linear trend $[F(1,31)=10.48$, $\mathrm{MSe}=188.61]$.

\section{GSR}

Skin resistance responses with latency between 1 and $5 \mathrm{sec}$ following stimulus onset were submitted to the ANOVA described above. Means are displayed against trial block and stimulus type in Figure 6. There were no significant differences between warning and imperative stimuli for either targets or nontargets. There was a larger response for targets vs. nontargets over both warning and imperative signals $[\mathrm{F}(1,31)=14.97, \mathrm{MSe}=.977]$. There was also some overall habituation indicated by a linear trend over trials $[\mathrm{F}(1,31)=6.58, \mathrm{MSe}=.883]$. Furthermore, responses to the target habituated more slowly than did responses to the target warning signal $[F(1,31)=$ 4.57, $\mathrm{MSe}=.331]$.
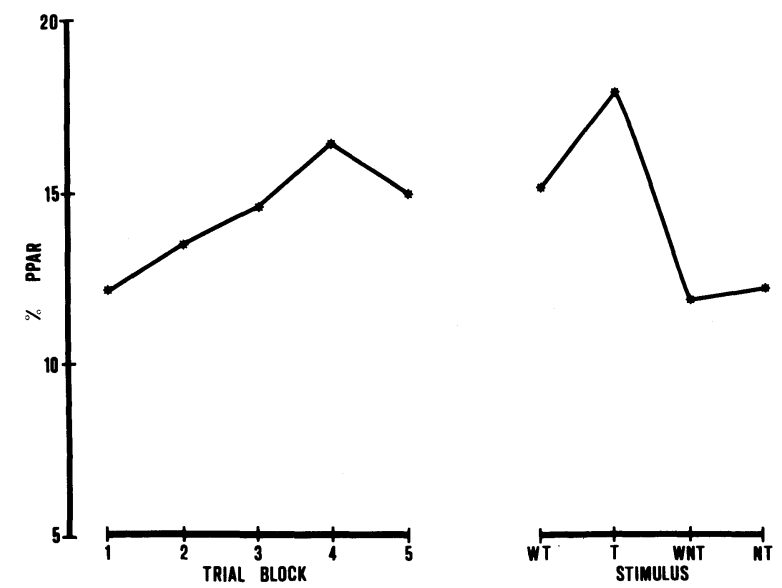

Figure 5. PPAR magnitude vs. trial block and stimulus type.
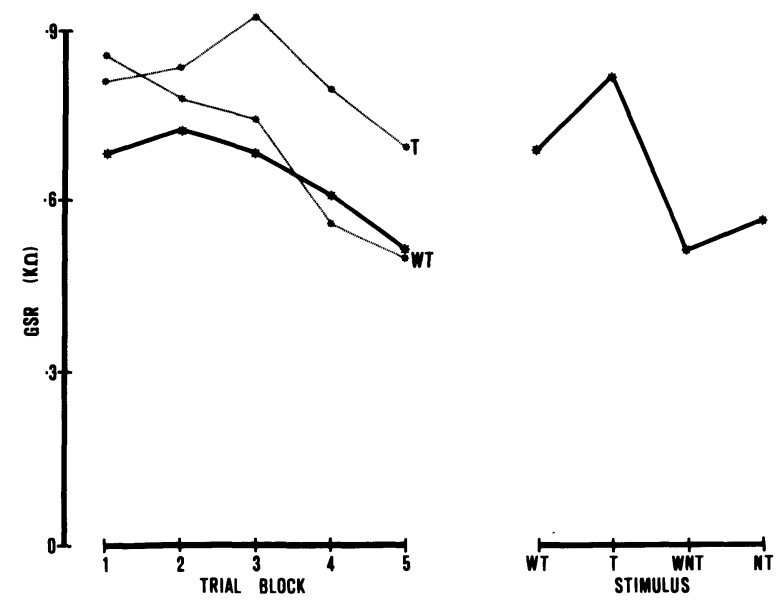

Figure 6. The effects of trial block and stimulus type upon GSR magnitude. 


\section{DISCUSSION}

The HR data indicate that the desired manipulation of cardiac arousal level occurred, with labeled warning signals producing an anticipatory increase in arousal before the target and a decrease before the nontarget. The effects of these arousal changes on PPAR, the focus of this study, were not as simple as expected. Rather, the effects predicted and obtained for the imperative stimuli were also obtained with the warning stimuli. That is, rather than the simple difference predicted between T and NT, PPARs obtained for both WT and T were larger than those obtained for WNT and NT. One possible explanation for this immediate arousal effect is apparent in the long latency of the PPAR, which extended to 12 beats poststimulus (approximately $9 \mathrm{sec}$ on the average). This would allow considerable time for arousal changes produced by a warning signal to influence the PPAR to that stimulus. When this experiment was designed, the expectation was that the anticipatory arousal changes would develop later in the ISI period, but it now appears that this expectation was unwarranted. Further research on this question is indicated. Aside from this, the results confirm the hypothesis that PPAR reflects arousal level, supporting the connection between PPAR and the S process of dual-process theory proposed by this author. That is, the magnitude register of the model of preliminary processes in OR elicitation shown in Figure 1 appears to reflect the coding of stimulus energy in a manner compatible with the sensitization process of Groves and Thompson (1970): through changes in state.

This sensitivity of PPAR to arousal level changes may clarify the causes of inconsistent results with habituation studies of this variable. While this laboratory has consistently failed to obtain habituation with PPAR, Furedy and his colleagues have found inconsistent results even with replications in the one laboratory (Furedy \& Arabian, 1979). The present results suggest that any systematic change in arousal level over trials would affect the magnitude of PPAR over trials. Furthermore, relatively slight HR changes were associated here with relatively large PPAR effects, indicating that PPAR is very sensitive to changes in state. Thus, a relatively slight relaxation over the course of an experiment might result in significant PPAR decrement, which could be construed as habituation. Obviously, further research with careful monitoring of state variables will be required to establish whether this hypothesis is correct.

Other aspects of the theory of preliminary processes in OR elicitation are supported by the fractionation of response measures obtained here. Specifically, the ECR, considered as an indicant of stimulus registration, was found, as predicted, to be independent of the experimental manipulations employed. In addition, respiratory pause was found to habituate rapidly, as required of a novelty indicator consistent with the hypothetical $\mathrm{H}$ process of Groves and Thompson (1970), and not to be dependent upon stimulus type.

The results for the GSR, considered as an OR index, are also of interest. Sokolov's theory would predict enhanced response magnitude for both the warning and imperative signals for the target in comparison with the nontarget. This prediction rests only on the greater signal value associated with the target. Dual-process theory would make the same prediction on the basis of the levels of S indicated by the PPAR results. The results are compatible with this prediction. Furthermore, both theories would predict a slower habituation of the response to the imperative target stimulus: Sokolovian theory because of the differential significance of the imperative compared with the warning signal, and dual-process theory because of the differential sensitization reflected in the PPARs to the two stimuli. Again, this prediction was supported by the data.

To date, the studies carried out in this laboratory have not tested the relative validity of these competing theories of OR elicitation and habituation. It must, however, be emphasized that dual-process theory is the only extant theory which can accommodate some aspects of the fractionation of phasic responses consistently reported from this laboratory and increasingly found elsewhere. In this context, the present results confirm the linkages between the operation of my novelty and magnitude registers and the $\mathrm{H}$ and $\mathrm{S}$ processes (respectively) of dual-process theory. That is, they support the identification of preliminary processes in OR elicitation, allowing the claim of "lawfulness" rather than "misbehavior" in the fractionation of phasic responses to OR-eliciting stimuli.

\section{REFERENCE NOTE}

1. Barry, R. J., \& Maltzman, I. Heart rate deceleration is not an orienting reflex; heart rate acceleration is not a defensive reflex. Manuscript submitted for publication, 1982.

\section{REFERENCES}

BARry, R. J. Physiological changes in a reaction-time task: Further problems with Sokolov's dimension of stimulus "significance." Physiological Psychology, 1978, 6, $438-444$.

BARRY, R. J. A factor-analytic examination of the unitary OR concept. Biological Psychology, 1979, 8, 161-178.

BARRY, R. J. Comparability of EDA effects obtained with constant current skin resistance and constant voltage skin conductance methods. Physiological Psychology, 1981, 9, 325-328. (a)

BARRY, R. J. Signal value and preliminary processes in OR elicitation. Pavlovian Journal of Biological Science, 1981, 16, 144-150. (b)

BARRY, R. J. Novelty and significance effects in the fractionation of phasic OR measures: A synthesis with traditional OR theory. Psychophysiology, 1982, 19, 28-35. 
BARRY, R. J., \& JAMES, A. L. Fractionation of phasic responses in a dishabituation paradigm. Physiology \& Behavior, 1981, 26, 69-75. (a)

BARRY, R. J., \& JAmEs, A. L. Fractionation of respiratory and vascular responses with simple visual stimulation. Physiological Psychology, 1981, 9, 96-101. (b)

Furedy, J. J., \& Arabian, J. M. A Pavlovian psychophysiological perspective on the OR: The facts of the matter. In H. D. Kimmel, E. H. van Olst, \& J. H. Orlebeke (Eds.), The orienting reflex in humans. Hillsdale, N.J: Erlbaum, 1979.

Ginsberg, S., \& Furedy, J. J. Stimulus repetition, change and assessments of sensitivities of and relationships among an electrodermal and two plethysmographic components of the orienting reaction. Psychophysiology, 1974, 11, 35-43.

Graham, F. K., \& Clifton, R. K. Heart-rate change as a component of the orienting response. Psychological Bulletin, 1966, 65, 305-320.

Groves, P. M., \& Thompson, R. F. Habituation: A dual-process theory. Psychological Review, 1970, 77, 419-450.

LACEY, J. I., \& LACEY, B. C. Verification and extension of the principle of autonomic response stereotypy. American Journal of Psychology, 1958, 71, 50-73.

Maltzman, I. Orienting reflexes and significance: A reply to O'Gorman. Psychophysiology, 1979, 16, 274-282.

Maltzman, I., \& Raskin, D. C. Effects of individual differences in the orienting reflex on conditioning and complex processes. Journal of Experimental Research in Personality, 1965, 1, 1-16.

O'Gorman, J. G. The orienting reflex: Novelty or significance detector? Psychophysiology, 1979, 16, 253-262.
Poor, D. D. S. Analysis of variance for repeated measures designs: Two approaches. Psychological Bulletin, 1973, 80, 204-209.

Sokolov, E. N. The higher nervous activity and the problem of perception. Acta Psychologica, 1955, 13, 134-135.

Sokolov, E. N. Neuronal models and the orienting reflex. In M. A. Brazier (Ed.), The central nervous system and behavior. Transactions of the 3rd conference. New York: Macey, 1960.

Thompson, R. F., Berry, S. D., Rinaldi, P. C., \& Berger, T. W. Habituation and the orienting reflex: The dual-process theory revisited. In H. D. Kimmel, E. H. van Olst, \& J. H. Orlebeke (Eds.), The orienting reflex in humans. Hillsdale, N.J: Erlbaum, 1979.

\section{NOTES}

1. A recent study in this laboratory (Barry, 1981a) found that stimulus-response relationships (such as habituation rate) obtained with these measures of the GSR were comparable to those obtained using rigorous constant-voltage skin conductance techniques with isotonic electrolyte, at least within OR-compatible stimulus ranges.

2. There was little variation in the reported totals, and this variable was not formally analyzed.

3. Significance level adopted in this report is $\mathrm{p}<.05$.

(Manuscript received December 8, 1981; revision accepted for publication March 30, 1982.) 